Niniejsza publikacja jest dostępna na licencji Creative Commons. Uznanie autorstwa-Użycie niekomercyjne-Bez utworów zależnych 3.0 Polska. Pewne prawa zastrzeżone na rzecz autora. Zezwala się na wykorzystanie publikacji zgodnie z licencja - pod warunkiem zachowania niniejszej informacji licencyjnej oraz wskazania autora jako właściciela praw do tekstu. Treść licencji jest dostęna na stronie: http://creativecommons.org/licenses/by-nc-nd/3.0/pl/

Lingwistyka Stosowana 18: 3/2016, 81-90

\author{
Magdalena OLPIŃSKA-SZKIEŁKO \\ Uniwersytet Warszawski
}

\title{
Anthropozentrische Sprachtheorie und Fachsprachenforschung
}

\begin{abstract}
:
Anthropocentric Theory of Language and Research into Languages for Special Purposes

The objective of the article is to review and to reconsider some significant achievements of the language for special purposes research in the light of the anthropocentric language theory by F. Grucza. In the first part the anthropocentric language theory is discussed in connection with the LSP research. The second part touches upon three most important problems of the LSP research, namely: 1) the question of the definition of the language for special purposes, 2) the issue of the separation of LSP and LGP and 3) the matter of specific features of LSP. First, the classic definitions of LSP as 1) linguistic system, 2) a means of communication and 3) the terminology will be discussed. Then, special attention will be focused on German legal language as an example of LSP. In the last part the author of the article will propose a definition of LSP in accordance with the anthropocentric language theory and will also try to solve the problem of the separation of the LSP and LGP, also of different LSPs from one another. The classic model of the vertical and the horizontal division of LSPs will be discussed, too.
\end{abstract}

\section{Einleitung}

In dem folgenden Beitrag wird versucht, die wichtigsten bisherigen Forschungsergebnisse im Bereich Fachsprachenlinguistik im Lichte der anthropozentrischen Sprachtheorie kritisch zu betrachten und zu revidieren.

Im ersten Teil der Ausführungen wird die anthropozentrische Sprachtheorie in ihren Grundzügen und in Bezug auf die Fachsprachenforschung dargestellt.

Im zweiten Teil werden die Schwerpunkte der Überlegungen auf folgende klassische Fragestellungen der Fachsprachenforschung gelegt:

1. Die Frage nach der Definition der Fachsprache;

2. Die Frage nach der Abgrenzung der Fachsprache von der Gemeinsprache;

3. Die Frage nach besonderen Merkmalen von Fachsprachen.

Zuerst werden die klassischen Definitionen der Fachsprachen zusammengestellt und im Lichte der anthropozentrischen Sprachtheorie diskutiert. Vor allem konzentriere ich mich auf folgende Forschungsperspektiven:

- Fachsprache als linguistisches System,

- Fachsprache als Kommunikationsmittel,

- Fachsprache als Terminologie.

Dabei wird die Rechtssprache als Beispiel einer Fachsprache unter die Lupe der anthropozentrischen Forschung genommen. Danach wird ein Versuch unternommen, 
eine anthropozentrische Definition der Fachsprache vorzuschlagen sowie das Problem der Abgrenzung einer Fachsprache von anderen Fachsprachen sowie von der Gemeinsprache zu lösen, das in der Fachsprachenforschung immer wieder auf Schwierigkeiten trifft (vgl. P. Sandrini 1996: 12ff.). Es wird auch auf die Problematik der horizontalen Gliederung und der vertikalen Schichtung der Fachsprachen eingegangen. Der folgende Text stellt eine überarbeitete und erweiterte Version des Beitrags dar, der auf dem XIII. Kongress der Internationalen Vereinigung für Germanistik (IVG) „Germanistik zwischen Tradition und Innovation“" in Shanghai, China, am 24.8.2015 vorgetragen wurde.

\section{Die anthropozentrische Sprachtheorie}

Die anthropozentrische Sprachtheorie, in früheren Beiträgen ihres Schöpfers die relativistische Theorie der menschlichen Sprachen genannt, wurde von Professor Franciszek Grucza, einem der bedeutendsten polnischen Sprachwissenschaftlern, und dem IVG-Präsidenten in den Jahren 2005-2010, erarbeitet (u.a. 1983, 1988, 1993a/b, 1997, 2005, 2007, 2012). Diese Theorie hatte einen großen Einfluss auf die Arbeiten anderer polnischer Linguisten - einen Überblick über diese Arbeiten bietet Sambor Grucza (2004: 38).

\subsection{Die Sprache als Kommunikationsfähigkeit}

Die anthropozentrische Sprachtheorie betrachtet die Sprachen vor allem als Idiolekte, als die individuellen Sprachen konkreter Menschen. Der Idiolekt ist die einzige Form der Sprache, die real existiert. Diesen individuellen realen Sprachen stellt die anthropozentrische Sprachtheorie die Polylekte gegenüber, die als logische Summen bzw. logische Schnitte der Idiolekte von Mitgliedern verschiedener Gemeinschaften (z.B. Nationen, Alters- oder Berufsgruppen) aufgefasst werden und die nur als linguistische Konstrukte (linguistische Modelle), also nicht wirklich, existieren.

Im Lichte der anthropozentrischen Sprachtheorie wird die Sprache - der Idiolekt als eine bestimmte Eigenschaft des Menschen definiert, als seine Fähigkeit zu kommunizieren, d.h. sprachliche Äußerungen zu produzieren, sie gezielt im Kommunikationsprozess als Kommunikationsmittel einzusetzen sowie sprachliche Äußerungen anderer Menschen zu empfangen und zu interpretieren (vgl. F. Grucza 1993a: 157; 1993b: 31).

Die Kommunikationsfähigkeit des Menschen lässt sich als eine Reihe von Teilkompetenzen darstellen. Sie lässt sich z.B. traditionell in 4 Sprachfähigkeiten: Sprechen, Schreiben, Hören/Hörverstehen und Lesen/Leseverstehen gliedern. Die einzelnen Sprachfähigkeiten können weiter in sprachliche (,rein“ sprachliche), diskursive, pragmatische und soziolinguistische Kompetenzen aufgeteilt werden, etwa so wie die folgende Tabelle 1 am Beispiel der Sprechfähigkeit zu verdeutlichen versucht: 


\begin{tabular}{|c|c|}
\hline $\begin{array}{l}\text { Sprechen/ } \\
\text { Sprechfähigkeit }\end{array}$ & $\begin{array}{l}\text { Linguistische (sprachliche) Kompetenz: } \\
-\quad \text { lexikalische Kompetenz, } \\
-\quad \text { grammatische Kompetenz (Fähigkeit, Äußerungen zu bilden, die } \\
\text { sinnvoll, kommunikativ adäquat und grammatisch = grammatika- } \\
\text { lisch korrekt sind), } \\
-\quad \text { semantische Kompetenz, } \\
-\quad \text { Aussprache - phonetisch-phonologische Kompetenz. } \\
\text { Diskursive Kompetenz: } \\
-\quad \text { Fähigkeit, sprachliche Äußerungen zu organisieren, zu strukturie- } \\
\text { ren und zu arrangieren; Fähigkeit, Äußerungen zu bilden, die } \\
\text { sinnvoll, kohärent, logisch und kommunikativ adäquat sind. } \\
\text { Pragmatische Kompetenz: } \\
-\quad \text { Fähigkeit, Kommunikationsabsichten auszudrücken, } \\
-\quad \text { interaktive Fähigkeiten. } \\
\text { Soziolinguistische Kompetenz: } \\
-\quad \text { Fähigkeit, Äußerungen gemäß den sozialen (kulturellen) Normen } \\
\quad \text { zu bilden. }\end{array}$ \\
\hline
\end{tabular}

Die kommunikative Fähigkeit eines Menschen wird durch sein umfangreiches Wissen begründet, dessen Struktur äußerst komplex ist (vgl. F. Grucza 1997: 12ff.). Vor allem handelt es sich um das sprachliche und nichtsprachliche Wissen des Menschen. Das sprachliche Wissen umfasst nach F. Grucza (ibid.: 16) u.a. semantisches und pragmatisches Form-, Funktions- und Realisationswissen. Das semantische Wissen ,berührt direkt, überlappt sich zu einem gewissen Grad mit dem Weltwissen“, und das pragmatische Wissen ,,ist mit dem kulturellen Wissen eng verbunden“ (ibid.: 17, übers. MOS). Das Weltwissen, das kulturelle Wissen und auch das Fachwissen sind Komponenten des nichtsprachlichen Wissens eines Menschen.

Aus diesem Versuch, das die menschliche Kommunikationsfähigkeit fundierende Wissen zu klassifizieren, folgt, dass „es keine klar definierte Grenze zwischen dem sprachlichen und dem nichtsprachlichen Wissen des Menschen gibt" (F. Grucza 2005: 47, übers. MOS). Um erfolgreich zu kommunizieren, muss der Mensch im Kommunikationsprozess nicht nur auf sein sprachliches, sondern auch auf sein nichtsprachliches Wissen zurückgreifen. Die Kommunikationsfähigkeit des Menschen kann dementsprechend als seine Fähigkeit charakterisiert werden, das sprachliche und nichtsprachliche Wissen in Kommunikationssituationen anzuwenden, d.h. beim Ausführen von Kommunikationsakten (sowohl bei der Produktion als auch bei der Rezeption von sprachlichen Äußerungen) auf seine Wissensbestände zurückzugreifen und die entsprechenden sprachlichen Formen auszuwählen, sie zu einer sinnvollen, kohärenten und logischen Einheit zusammenzufügen sowie der Äußerung eine individuelle (z.B. emotionale) Färbung hinzuzufügen (oder sie zu interpretieren).

Das Wissen, das die menschliche Kommunikationsfähigkeit begründet, kann nicht direkt übertragen werden. Jeder Mensch kann dank Erfahrung, Praxis oder Reflexion sein Wissen gewinnen (erarbeiten), sammeln, entwickeln, verarbeiten und auch in seinen sprachlichen Äußerungen mit Hilfe von sprachlichen Formen veräußerlichen, repräsentieren (F. Grucza 1997: 13; vgl. auch 1993b: 27). Aufgrund dieser äußeren Repräsentationen muss jeder andere Mensch sein eigenes Wissen selbst (re)konstruieren. 


\subsection{Sprachliche Äußerungen als Kommunikationsmittel}

Weder Idiolekte noch Polylekte lassen sich direkt einer linguistischen Untersuchung unterziehen, denn die einzigen der menschlichen Wahrnehmung und somit auch der Forschung zugänglichen Repräsentationen des menschlichen (sprachlichen und nichtsprachlichen) Wissens sind sprachliche Äußerungen, die im Kommunikationsprozess verwendet werden. Im Kommunikationsprozess kommen sprachliche Äußerungen in strukturell geordneter Form als Texte (Diskurse) vor, in denen mit Hilfe von verbalen Ausdrücken die Textproduzenten ihre Kommunikationsabsichten und gewünschten Inhalte kodieren. Aufgrund der Kenntnis von verbalen Ausdrücken, deren Auswahl und Organisation in der Äußerung durch die Kommunikationssituation und den Kontext determiniert werden (vgl. N. Fries 2005: 153) - denn sprachliche Äußerungen, die als Kommunikationsmittel eingesetzt werden, sind stets unmittelbar mit der gegebenen Situation verbunden und in den Kommunikationskontext eingebettet - können die Empfänger die Äußerung dekodieren und ihr die vom Textproduzenten beabsichtigten Bedeutungen und Inhalte zuweisen.

Nun, in welcher Beziehung steht die anthropozentrische Sprachtheorie zur Fachsprachenforschung?

\section{Fachsprache - Versuch einer Definition}

\subsection{Fachsprache als linguistisches System oder als Kommunikationsmittel?}

Den Versuch, diese Frage zu beantworten, beginne ich mit der klassischen Definition von L. Hoffman (1985: 53), der die Fachsprache als „die Gesamtheit aller sprachlichen Mittel, die in einem fachlich begrenzbaren Kommunikationsbereich verwendet werden, um die Verständigung zwischen den in diesem Bereich tätigen Menschen zu gewährleisten" bezeichnet.

$\mathrm{Ob}$ die Gesamtheit der sprachlichen Mitteln als eine bestimmte „Sammlung“/ Summe (also als linguistisches System) oder als eine geordnete Menge von sprachlichen Mitteln (also als die der Verständigung dienenden sprachlichen Äußerungen) begriffen werden sollte, bleibt unklar, denn einerseits wird in der Fachliteratur von der Fachsprache als linguistischem System gesprochen, doch andererseits werden ihr Merkmale zugeschrieben, die sich eindeutig auf sprachliche Äußerungen beziehen. Dies geht deutlich aus den Aussagen von J. Gebhard (1987: 23) oder D. Möhn und R. Pelka (1984: 26) hervor:

Fachsprachen sind keine eigenständigen, von der Allgemeinsprache abgehobenen linguistischen Systeme, sondern Teilmengen der Gesamtsprache, die durch die Auswahl und die Frequenz bestimmter sprachlicher Erscheinungsformen (z.B. auf lexikalischer, syntaktischer und morphologischer Ebene) gekennzeichnet sind (J. Gebhard 1987: 23; Unterstreichungen MOS) und

Sprachsystembezogen kann Fachsprache als ein Subsystem dargestellt werden, das durch eine spezifische Auswahl und Nutzung sprachlicher Mittel in morphologischer, lexikalischer, syntaktischer und textlicher Hinsicht charakterisiert ist. (D. Möhn/ R. Pelka 1984: 26; Unterstreichungen MOS). 
Die meisten Fachsprachenforscher neigen jedoch dazu, wie es scheint, die Fachsprache als eine geordnete Menge (als Kommunikationsmittel) anzusehen.

Fachsprachen sind z.B. nach W. Schmidt (zit. nach H.-R. Fluck 1991: 14; Unterstreichung MOS), ,das Mittel einer optimalen Verständigung über ein Fachgebiet unter Fachleuten. Sie ist gekennzeichnet durch einen spezifischen Wortschatz und spezielle Normen für die Auswahl, Verwendung und Frequenz gemeinsprachlicher lexikalischer und grammatischer Mittel“". Auch P. Sandrini (1996, 13), einer der bedeutendsten Rechtsspracheforscher, bezeichnet die Fachsprache als Kommunikationsmittel ,zwischen Fachleuten untereinander und zwischen Fachleuten und Laien innerhalb eines definierten Fachbereichs“. Auch er gibt zu (ibid.), dass „dieses Verständigungsmittel durch einen spezifischen Fachwortschatz und spezielle Normen für die Auswahl, Verwendung und Frequenz gemeinsprachlicher lexikalischer und grammatischer Mittel“" gekennzeichnet ist.

L. Hoffmann (1989, 14f.), der sich ebenfalls mit der Fachsprache des Rechts beschäftigt, identifiziert eine Reihe von Merkmalen, mit denen die Rechtsprache ,ausgestattet ist":

- ein Inventar rechtlicher Termini, zu denen alltagssprachliche Ausdrücke mit modifizierter, spezieller Bedeutung [...], Archaismen und Formeln [...] gehören;

- eine extensive Nutzung syntaktischer Möglichkeiten der Gegenstandsbestimmung (Attributhäufung, oft mit Präpositionalgruppen, Relativsätze), um das erwünschte Maß an Präzision zu erreichen;

- weitgehender Verzicht auf Anapher und Weglassung zugunsten referentieller Eindeutigkeit;

- Verwendung generalisierter Ausdrücke zur Angabe von Geltungsbereichen;

- Realisierung von (...) komplexen Propositionen, um Tatbestände hinreichend genau zu charakterisieren;

- konditionale Konstruktionen und Negationen zum Ausdruck von Einschränkungen und Bedingungen;

- Gebrauch unpersönlicher Ausdrücke (Passiv, Indefinitpronomen etc.) [...]. (ibid. Unterstreichungen MOS).

Aus dieser Darstellung geht deutlich hervor, dass hier die Fachsprache (des Rechts) mit dem (Rechts)Text gleichgesetzt wird. Die oben angeführten Charakteristika beziehen sich nämlich auf Rechtstexte und nicht auf das (Fach)Wissen, das die Produktion dieser Texte ermöglicht. Die Identifikation der (Fach)Sprache und des (Fach)Textes ist manifest ebenso bei anderen Fachsprachenforschern, wie z.B. bei P. Roessler, der die österreichische Rechtssprache, insbesondere die ,geschriebene Rechtssprache“" (1994: 35; Unterstreichung MOS) zum Objekt seiner linguistischen Untersuchung macht, mit der Absicht festzustellen, „ob sich Syntax und Stil der österreichischen Rechtssprache [seit dem ausgehenden 18. Jahrhundert] verändert haben“"(ebd.: 37). Seine Analyse betrifft u.a.:

- die Verteilung von Einfachsätzen, Satzgefügen, Satzreihen und Aufzählungen [in Rechtstexten],

- Anzahl der Nebensätze im Satzgefüge,

- Verteilung des Aktivs und Passivs, 
- Verteilung der Hilfs- und Modalverben,

- den Anteil der Nomen und Pronomen an der Gesamtheit der Wörter usw.

Unmissverständlich stellt P. Roessler die Identität der Rechtssprache und des Rechtstextes fest, wenn er folgendes bemerkt: „Bekanntlich besteht die 'besondere Leistung' der Rechtssprache darin, einen 'trockenen', 'abstrakten', 'unpersönlichen', 'sperrigen', 'umständlichen', 'monotonen', 'schwierigen', Stil zu haben“ (ebd.: 34).

Im Lichte der anthropozentrischen Sprachtheorie, die die Sprache als menschliche Kommunikationsfähigkeit und die Texte als Produkte der menschlichen Kommunikationstätigkeit scharf unterscheidet, ist diese Gleichsetzung unmöglich anzunehmen. Die anthropozentrische Sprachtheorie lehnt sowohl die Begriffsbestimmung der Fachsprache als Kommunikationsmittel wie auch als linguistisches System ab und identifiziert die sprachlichen Äußerungen (Texte) als die Verständigungsmittel der Menschen.

Darüber hinaus könnte man bemerken, dass sich aus der Gleichsetzung von Fachsprache und Fachtext als Konsequenz auch noch die Unausführbarkeit einer genaueren Trennung der Fachsprache von der Gemeinsprache (und von anderen Fachsprachen ebenfalls) ergibt, denn, wie es beispielsweise P. Sandrini bemerkt, die Fachsprache (in seinem Fall die Rechtssprache) ,ist mit der Gemeinsprache sehr eng verbunden und enthält sehr viele gemeinsprachliche Elemente" (ibid.: 15).

Eine plausiblere Möglichkeit einer Abgrenzung der Fachsprache von der Gemeinsprache bietet die in der Fachsprachenforschung als überholt geltende (vgl. u.a. P. Sandrini 1996: 15) „Auffassung der frühen Terminologieforschung, Fachsprache sei eine terminologische Erweiterung der Gemeinsprache“.

Wie verhält sich die anthropozentrische Sprachforschung zu dieser Auffassung?

\subsection{Fachsprache als Terminologie}

Wenn wir für einen kurzen Moment annehmen würden, dass die Fachsprache mit der Terminologie eines Faches identisch wäre, so könnten wir die Fachsprache als die Gesamtheit aller Termini eines bestimmten Fachbereichs bezeichnen. Die Bestandteile der Termini sind Benennungen, d.h. einfache und komplexe sprachliche Bezeichnungen (nicht sprachliche Bezeichnungen, wie Symbole, Nummern und Formeln werden an dieser Stelle außer Acht gelassen), und Begriffe (Begriffssysteme), die semantisches Wissen und Fachwissen umfassen:

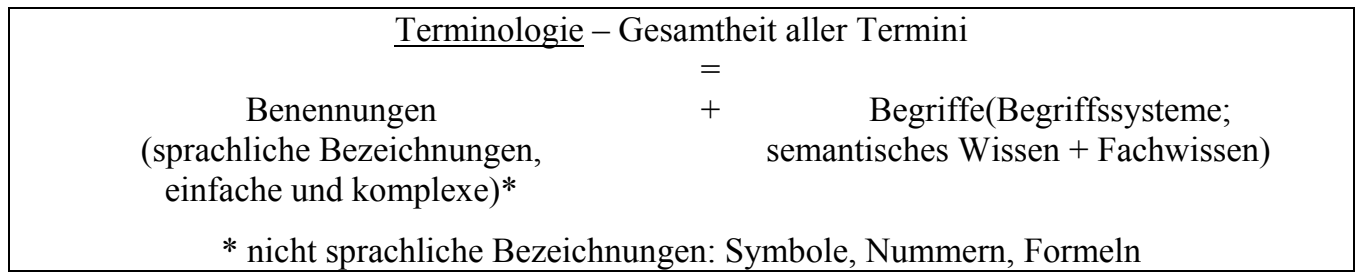

Terminologien jeweiliger Fachbereiche bilden ,spezialisierte Datenbanken“, die komplexes Fachwissen repräsentieren (vgl. F. Grucza 2002: 15). Diese Auslegung der Terminologie als Fachwissen entspricht weitgehend der anthropozentrischen Auffassung der Sprache als Wissenskomplex, das die menschliche Kommunikationstätigkeit begründet: 


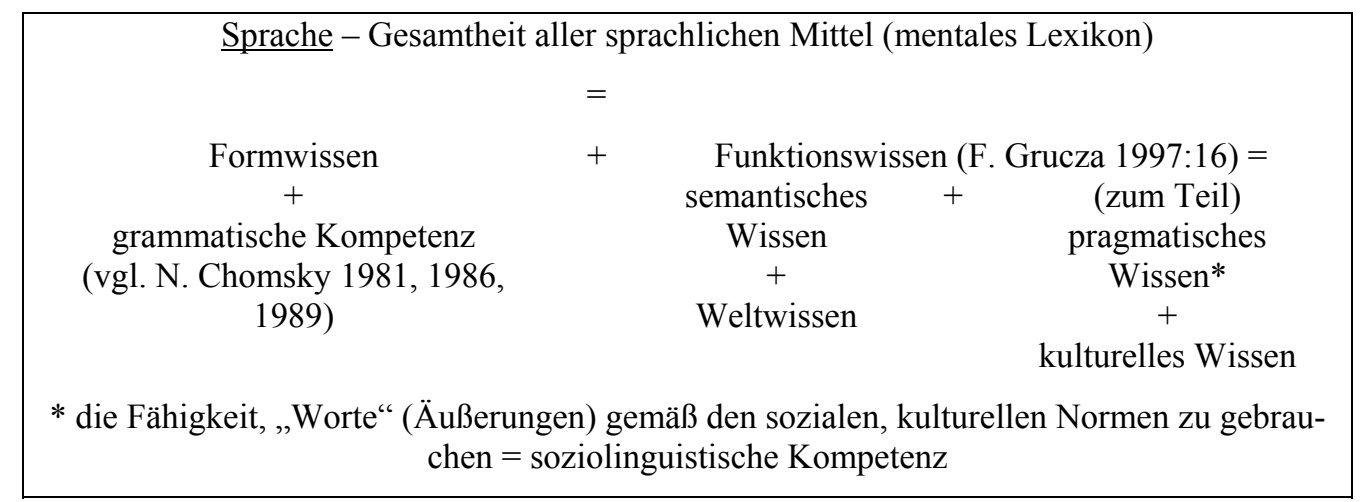

Die (Gemein)Sprache als Wissenskomplex umfasst nach F. Grucza (1997: 16), wie oben erwähnt, das Wissen über die Formen von Morphemen, Lexemen, Phrasemen usw. sowie das Wissen über ihre Funktionen, über ihre Bedeutung. Dem Formwissen kann das grammatische Wissen hinzugefügt werden, so wie es z.B. N. Chomsky (1981, 1986, 1989) begriffen hat (grammatische Kompetenz: lexikalische Elemente verfügen über bestimmte Eigenschaften, die sie bestimmte syntaktische Relationen mit anderen lexikalischen Elementen eingehen lassen oder es ihnen verbieten), und dem semantischen und pragmatischen Funktionswissen - das allgemeine Weltwissen und (sozio)kulturelles Wissen (soziokulturelle Kompetenz).

Jedoch wenn wir diese Darstellung mit der im ersten Teil der Ausführungen angeführten anthropozentrischen Definition der Sprache vergleichen und unsere Aufmerksamkeit wieder kurz der an jener Stelle präsentierten Zusammenstellung der Kommunikationsteilkompetenzen schenken (Tabelle 1), so können wir feststellen, dass einige Elemente in der letzteren Darstellung fehlen. Es handelt sich vor allem um die Diskurskompetenz, die Fähigkeit, Kommunikationsabsichten auszudrücken und zu verstehen, und die interaktiven Fähigkeiten.

Daraus ergibt sich folgende Schlussfolgerung: Obwohl die Bestimmung der Fachsprache als Terminologie eines bestimmten Faches der anthropozentrischen Betrachtungsweise näher liegt als die Auffassung, die Fachsprachen seien linguistische Systeme oder Verständigungsmittel, kann sie nicht angenommen werden, genauso wie man die Sprache nicht mit dem mentalen Lexikon identifizieren kann. Bei der Beschreibung der Sprache/Fachsprache dürfen keine Elemente fehlen, die die gesamte menschliche Kommunikationsfähigkeit ausmachen.

Wie könnte also eine anthropozentrische Definition der Fachsprache lauten?

\subsection{Fachsprache im Lichte der anthropozentrischen Sprachtheorie}

Im Lichte der anthropozentrischen Sprachtheorie können wir, wie oben angeführt, die menschliche Sprache - den Idiolekt - als die Gesamtheit des sprachlichen Wissens eines Menschen definieren, das mit dem nichtsprachlichen Wissen eng verknüpft und verflochten ist. Demensprechend können wir den Fachidiolekt eines Menschen als die Gesamtheit seines fachsprachlichen Wissens definieren, das mit seinem nichtsprachlichen - fachlichen Wissen eng verknüpft und verflochten ist. Der Fachidiolekt bildet einen Teil des entsprechenden Idiolektes; es ist insbesondere die Terminologie (als ein 
Teil des gesamten Lexikons), aber auch zum Teil die diskursive Kompetenz (die Fähigkeit Fachäußerungen gemäß den fachspezifischen Anforderungen zu bilden). Es gibt keine klar definierte Grenze zwischen dem Idiolekt und dem Fachidiolekt, der wiederum als eine ,getrennte“ Größe ausschließlich ein linguistisches Konstrukt ist.

Weil es in Wirklichkeit keine Abgrenzung des Fachidiolektes eines Menschen von seinem gesamten Idiolekt gibt, können wir annehmen, dass der Mensch - der Spezialist - bei der Ausführung seiner Kommunikationshandlungen, d.h. bei der Produktion und Rezeption von fachbezogenen sprachlichen Äußerungen nicht nur auf seine fachsprachlichen Kompetenzen, sondern auf die gesamten Ressourcen seines Wissens zurückgreift. Daraus folgt, dass sich ein Fachwort nicht eindeutig aufgrund seiner Zugehörigkeit zur jeweiligen Fachsprache (Fachidiolekt) identifizieren lässt. Aus diesem Grund muss man annehmen, dass bei der Bestimmung, ob ein sprachliches Element ein Fachwort ist oder nicht, seine Zugehörigkeit zum Fachtext ausschlaggebend ist. Anthropozentrisch betrachtet, ist wiederum bei der Bestimmung des Fachtextes im Gegensatz zum Nicht-Fachtext sein Produzent entscheidend, der ein Fachmann sein muss, der sein fachliches Wissen mithilfe der jeweiligen Äußerung ausdrückt.

Darüber, dass die primäre Funktion des Fachtextes darin besteht, Fachwissen zu repräsentieren, wird in der Fachsprachenforschung nicht gestritten; vgl. z.B. P. Sandrini (1996: 4; Unterstreichungen MOS):

Durch eine besondere Auswahl von sprachlichen Möglichkeiten wird fachliches Wissen dargestellt und die Kommunikation zwischen Fachleuten ermöglicht. Erhebt man den Kommunikationsinhalt zum Unterscheidungskriterium, so lassen sich Texte jeder Art einer bestimmten Menge zuweisen; es lässt sich der Umriss einer bestimmten Sub- oder Fachsprache erkennen. (ibid.)

Was in dieser Aussage besondere Beachtung verdient, ist der Ansatz zur Abgrenzung verschiedener Fachsprachen voneinander, die auf der Ebene der Texte durchgeführt werden könnte. Und ebenfalls auf der Ebene der Texte lässt sich, anthropozentrisch betrachtet, die Grenze zwischen einer Fachsprache und der Gemeinsprache ziehen. Genaugenommen lassen sich Fachtexte als Produkte der Fachsprachen (Fachidiolekte) und Repräsentationen des fachlichen Wissens von Nicht-Fachtexten als Produkten der Gemeinsprache unterscheiden. Im Lichte der anthropozentrischen Sprachtheorie lassen sich auch lediglich sprachliche Äußerungen (Texte), und nicht (Fach)Sprachen vertikal schichten. Wenn man die vertikale Schichtung, so wie sie z.B. P. Sandrini (1996: 5; Unterstreichungen MOS) darstellt, unter die Lupe der anthropozentrischen Forschung nimmt, so erkennt man sofort, dass hier Charakteristika von Fachtexten, und nicht von (Fach)Sprachen angegeben werden:

A. 1. Höchste Abstraktionsstufe, 2. künstliche Symbole für Elemente und Relatio-

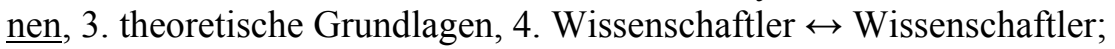
en Adaption übernommen, so konnten die einzelnen zu beachtenden Bereiche gut Sprache für Relationen (Syntax), 3. experimentelle Wissenschaften, 4. Wissenschaftler (Techniker) $\leftrightarrow$ Wissenschaftler (Techniker) $\leftrightarrow$ wissenschaftlichtechnische Hilfskräfte;

C. 1. hohe Abstraktionsstufe, 2. natürliche Sprache mit einem sehr hohen Anteil an Fachterminologie und einer streng determinierten Syntax, 3. angewandte Wissen- 
schaften und Technik, 4. Wissenschaftler (Techniker) $\leftrightarrow$ wissenschaftliche und technische Leiter der materiellen Produktion;

D. 1. niedrige Abstraktionsstufe, 2. natürliche Sprache mit einem hohen Anteil an Fachterminologie und einer relativ ungebundenen Syntax, 3. materielle Produktion, 4. wissenschaftliche und technische Leiter der materiellen Produktion $\leftrightarrow$ Meister $\leftrightarrow$ Facharbeiter (Angestellte);

E. 1. sehr niedrige Abstraktionsstufe, 2. natürliche Sprache mit einigen Fachtermini und ungebundener Syntax, 3. Konsumtion, 4, Vertreter der materiellen Produktion $\leftrightarrow$ Vertreter des Handels $\leftrightarrow$ Konsumenten $\leftrightarrow$ Konsumenten.

Und wie steht es um die anthropozentrische Betrachtungsweise der horizontalen Gliederung der Fachsprachen? Mit dieser Frage komme ich zum letzten Punkt meiner Ausführungen - zu der Fachsprache als Fachpolylekt.

\subsection{Fachsprache als Fachpolylekt}

(Fach)Polylekte können im Lichte der anthropozentrischen Sprachtheorie als logische Summen oder logische Schnittmengen von (Fach)Idiolekten verstanden werden. Sie sind - genauso wie Fachidiolekte als unabhängige Einheiten - linguistische Konstrukte. Ausschlaggebend für die Bestimmung eines Fachpolylektes ist die Existenz einer Gruppe von Menschen, die über vergleichbares, oder annährend gleiches Fachwissen verfügen. Wie D. Möhn und R. Pelka schon 1984 bemerkten (S. 26, Unterstreichung MOS), ist „,der Begriff ,Fachsprache“ primär auf die Gruppe ihrer Träger, die Fachleute zu beziehen“, im weiteren Sinne jedoch auch auf alle in dem gegebenen Fach Handelnden oder ,fachlich Interessierte“"،.

In diesem Sinne sollte man die klassische horizontale Gliederung der Fachsprachen von L. Hoffmann (hier: 1985) überdenken und revidieren. Als die Grundlage für die Gliederung sollte nicht die Verschiedenheit der Fachbereiche dienen (Technik, Medizin, Recht usw.), sondern die Beschaffenheit von unterschiedlichen Gruppen von Spezialisten. Die Aussage von J. Gebhard (1987: 23): „Es gibt keine Fachsprache an sich, sondern immer nur verschiedene Fachsprachen einzelner Fachdisziplinen“, sollte demnach umformuliert werden und lauten: Es gibt keine Fachsprache - keinen Fachpolylekt an sich, sondern nur verschiedene Fachsprachen - Fachpolylekte einzelner Expertengruppen.

\section{Bibliographie}

Chomsky, N. (1981), Lectures on Government and Binding. Dördrecht.

Chomsky, N. (1986), Barriers. Cambridge, MA.

Chomsky, N. (1989), Some notes on economy of derivation and representation. MIT Working Papers in Linguistics 10. Cambridge, 43-74.

Fluck, H.-R. (1991), Fachsprachen. Tübingen.

Fries, N. (2005), Textkompetenz: ein Essay über ausgewählte sprachspezifische und interkulturelle Aspekte. In: F. Grucza, et al. (Hrsg.), Germanistische Erfahrungen und Perspektiven der Interkulturalität. Warszawa, 150-161. 
Gebhard, J. (1987), Fachsprachlicher Begleitunterricht im Intensivsprachkurs. In: H.P. Kelz (Hrsg.), Fachsprache. Studienvorbereitung und Didaktik der Fachsprachen. Bonn, 23-34.

Grucza, F. (1983), Zagadnienia metalingwistyki. Warszawa.

Grucza, F. (1988), Zum Begriff der Sprachkompetenz, Kommunikationskompetenz und Kulturkompetenz. In: N. Honsza/ H.-G. Roloff (Hrsg.), Daß eine Nation die andere verstehen möge. Festschrift für Marian Szyrocki zu seinem 60. Geburtstag. Beihefte zu Daphnis Bd. 7. Amsterdam, 309-331.

Grucza, F. (1993a), Język, ludzkie właściwości językowe, językowa zdolność ludzi. In: J. Piontek/ A. Wiercińska (Hrsg.), Człowiek w perspektywie ujęć biokulturowych. Poznań, 151-174.

Grucza, F. (1993b), Zagadnienia ontologii lingwistycznej: o językach ludzkich i ich (rzeczywistym) istnieniu. In: Opuscula logopedica in honorem Leonis Kaczmarek. Lublin, 25-47.

Grucza, F. (1997), Języki ludzkie a wyrażenia językowe, wiedza a informacja, mózg a umyst ludzki. In: F. Grucza/ M. Dakowska (Hrsg.), Podejścia kognitywne w lingwistyce, translatoryce i glottodydaktyce. Warszawa, 7-21.

Grucza, F. (2005), Wyrażenie „upowszechnianie nauki” - jego status i znaczenie w świetle teorii aktów komunikacyjnych i lingwistyki tekstów. In: F. Grucza/ W. Wiśniewski (Hrsg.), Teoria i praktyka upowszechniania nauki wczoraj i jutro. Warszawa, 41-76.

Grucza, F. (2007), Lingwistyka stosowana. Historia - Zadania - Osiagnięcia. Warszawa.

Grucza, F. (2012), Antropocentryczna a paradygmatyczna (tradycyjna) lingwistyka (stosowana) $i$ kulturologia (stosowana). In: Lingwistyka Stosowana/ Applied Linguistics/ Angewandte Linguistik 6/2012, 5-44.

Grucza, S. (2004), Od lingwistyki tekstu do lingwistyki tekstu specjalistycznego. Warszawa.

Hoffmann, L. (1985), Kommunikationsmittel Fachsprache. Eine Einführung. Tübingen.

Hoffmann, L. (1989), Einleitung: Recht - Sprache - Diskurs. In: L. Hoffmann (Hrsg.), Rechtsdiskurse. Untersuchungen zur Kommunikation im Gerichtsverfahren. Tübingen, 9-38.

Möhn, D./ R. Pelka (1984), Fachsprachen. Eine Einführung. Tübingen.

Roessler, P. (1994), Entwicklungstendenzen der österreichischen Rechtssprache seit dem ausgehenden 18. Jahrhundert. Frankfurt a. M. etc.

Sandrini, P. (1996), Terminologiearbeit im Recht. Wien. 